\title{
Use of Color Histograms for Image Identification
}

\author{
Yuriy Furgala \\ department of electronic and computer technologies, \\ Ivan Franko National University of Lviv, \\ Lviv, Ukraine \\ furgala@mail.lviv.ua \\ Bohdan Rusyn \\ Karpenko Physico-Mechanical Institute of the NASU, \\ Lviv, Ukraine, \\ University of Technology and Humanities \\ Radom, Poland \\ rusyn@ipm.lviv.ua
}

\author{
Andriy Velhosh \\ department of electronic and computer technologies, \\ Ivan Franko National University of Lviv, \\ Lviv, Ukraine \\ andriyvelgosh@gmail.com \\ Yuriy Korchak \\ department of electronic and computer technologies, \\ Ivan Franko National University of Lviv, \\ Lviv, Ukraine \\ yurakorchak@yahoo.co.uk
}

\begin{abstract}
The paper analyzes the possibility of using color images' histograms formed in the color spaces $H S^{*}$ for images distinguishing by their content. It has been established that the use of $\boldsymbol{H}$-histograms allows you to successfully recognize an image when its size is changed 128 times and does not depend on the aspect ratio of the image.
\end{abstract}

Index Terms-color images, image's color histogram; images comparison.

\section{INTRODUCTION}

The identification of objects in images is carried out by a set of their characteristics, the nature of which is very different. In general, these features are divided into three levels: the bottom, which includes the color and texture; average contours and outlines; top - semantic and statistical characteristics [1-3]. Depending on the implementation of the method of obtaining attributes, this may be the geometric configuration of special points, the histogram of orientation of gradients, the set of spatial frequencies, and many others. The proper place among them is occupied by methods based on the analysis of histogram intensities both in monochrome version and in color. The role of the subject of research most often used histograms of colors in the system RGB, however, there are works that show the possibility of using for the identification of histogram images in color space $C I E L^{*} a * b *$ [4-7]. However, the closest to the spectral representation of color are systems that carry out a description of the color in a cylindrical coordinate system, where the color is an angular coordinate. A number of these systems involve at once three representatives of $H S L, H S V$ and $H S I$, each of which has certain features, however, in our opinion, may be suitable for color identification of objects in the image.

\section{RESULTS AND DISCUSSION}

The paper analyzes the possibility of color images histograms using on the case of forming a description of the color coordinates of pixels in the spaces of HSL, HSV and HSI. The common feature of these HSL, HSV and HSI color schemes is the method of determining the color coordinate $H$ (hue), which in all three systems has an angular coordinate in a cylindrical coordinate system and has content on the scale of color change from red to yellow to green, then through blue to blue and then through raspberry again to red. The coordinate $S$ (saturation) has saturation content and corresponds to the distance from the cylinder axis. Despite the common denominator, in all these color systems, $S$ is defined differently. The motion along the cylinder's axis corresponds to a change in the brightness $I$ (intensity), $V$ (value) or $L$ (lightness) from zero to maximum, that is, the change in color from dark to light due to the shades of the given color. It is obvious that the method of calculating these quantities in each system is different. Thus, in all these systems, $H$ has the content of the spectral composition of color, and the other two coordinates only determine its brightness and saturation. So the $H$-histogram can be used as a spectral identifier of whole image or an individual object on image.

Some datasets are popularly used for various purposes in the field of image investigation; these include the Corel dataset, the Brodatz texture dataset, the OUTex texture dataset, the Coil-100 dataset, the ETH-80 dataset, the Caltech 101 dataset and the PASCAL VOC dataset. Self-collected datasets can also be used for image retrieval. In our study, for the numerical assessment of the similarity of histograms, the images shown in Fig. 1 were used. in formats *.bmp, *.png and *.jpg as well as a set of 10 images from the Caltech 101 dataset [8]. The choice of formats is due to a fundamentally different way of storing information about the distribution of pixels brightness. The smallest linear size of output images was at least 1024 pixels. In the course of the experiments, it was found that the results for *.bmp and *.png formats are identical; therefore, the research data will be submitted only for one of them. 

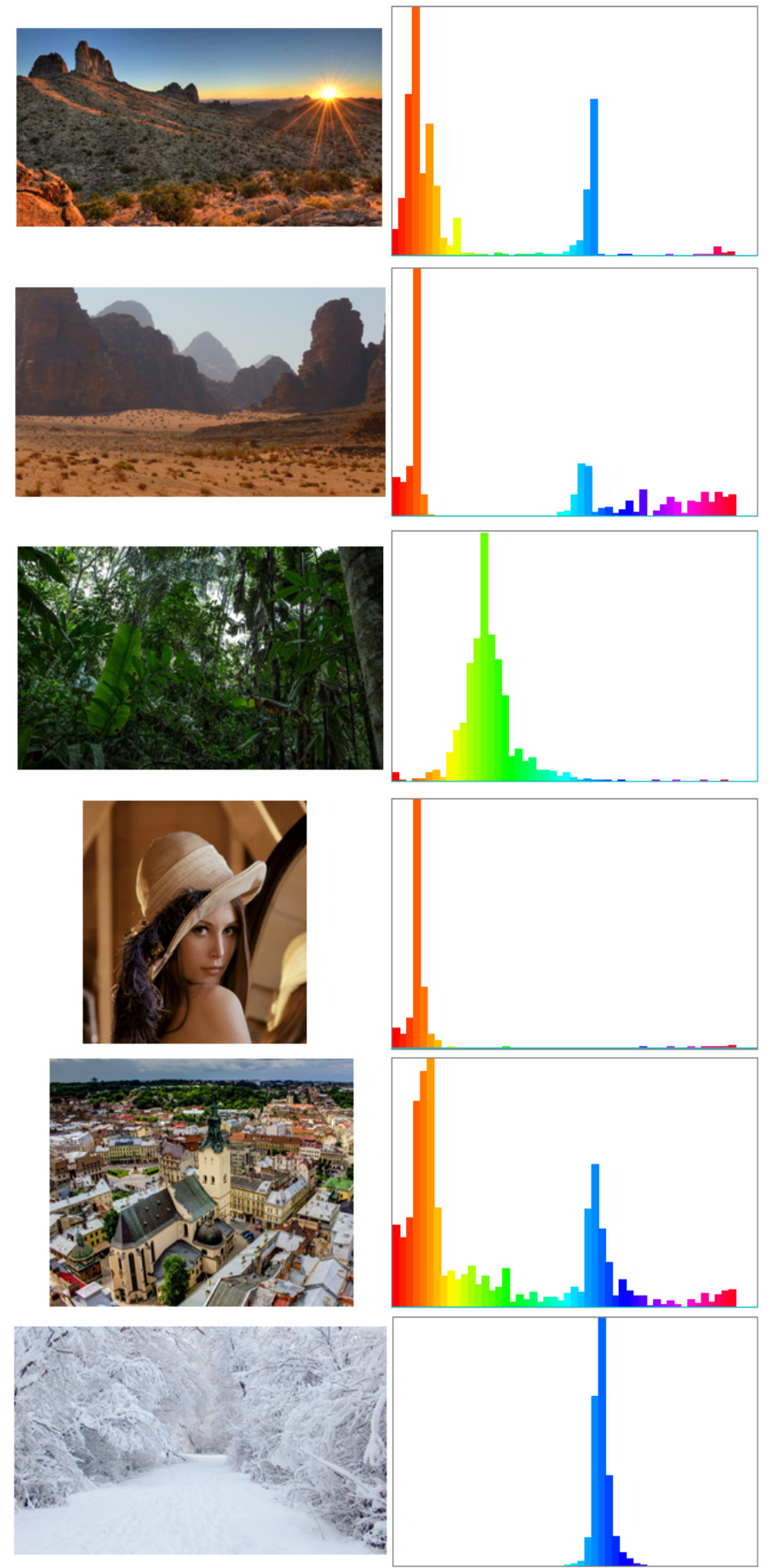

Figure 1. Test images and their $H$-histograms. Along the abscissa axis, the value of the color coordinate $\mathrm{H}$ is set aside within the full cycle of its change.
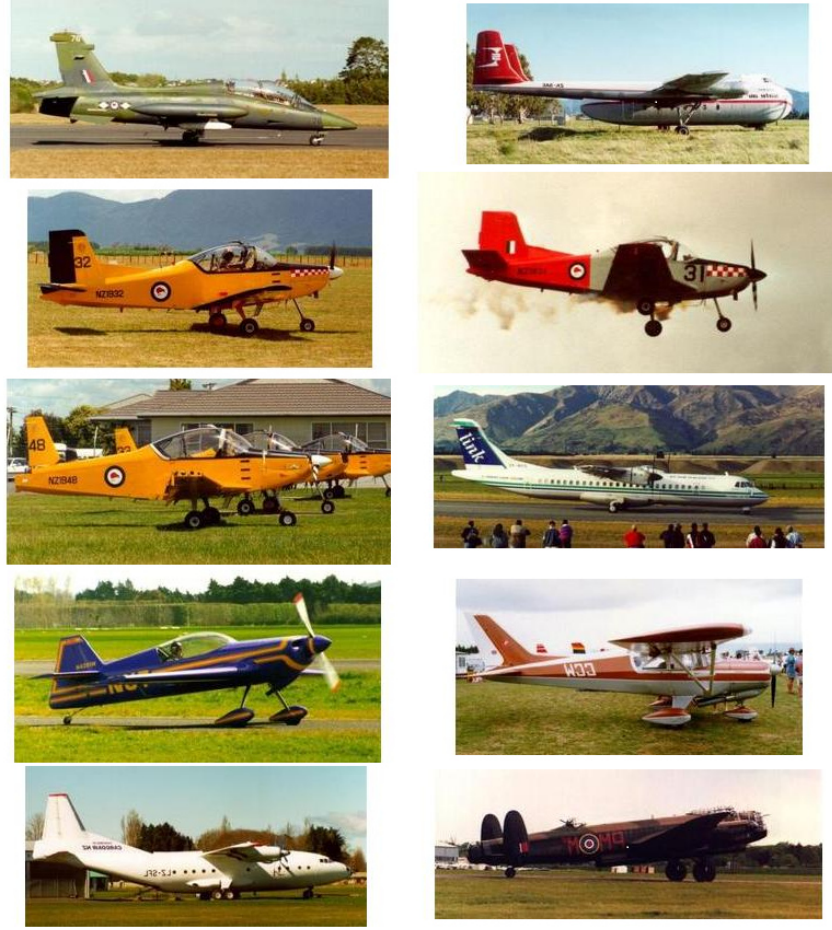

Figure 2. Investigated images from Caltech 101 dataset.

An assessment of the histograms similarity was carried out by calculating the cross-correlation coefficient $R$ :

$$
R=\frac{\iint_{A}\left(g(i)-G_{A}\right)\left(s(i-\xi)-S_{A}\right) d i}{\sqrt{\iint_{A}\left(g(i)-G_{A}\right)^{2} d i d j} \sqrt{\iint_{A}\left(s(i-\xi)-S_{A}\right)^{2} d i}},
$$

where $G_{A}=\iint_{A} g(i) d i, S_{A}=\iint_{A} s(i) d i, g(i)$ and $s(i)-H$ histograms of the corresponding images.

The obvious difference between histograms of different images gives confidence in obtaining numerically measured differences between the color characteristics of images or objects on them. The study of the set of own test images presented in Fig.1 and 10 of the standard images from the Caltech 101 dataset shows that the maximum value of the correlation coefficient for different images of the original size is 0.89 and is observed for the case of different angles of the same scenes. In other cases, the coefficient of cross correlation $R$ does not exceed 0.60 . That is, between the value of the crosscorrelation coefficient $R$ for identical and different images, there is a noticeable spar, which allows a reliable separation of objects.

However, when solving problems of object recognition, their size in the image, as a rule, differs from the size of the template, and sometimes, quite substantially. Hence the task of evaluating the limits of the use of $H$-histograms for comparing images in wide variations of their size. 

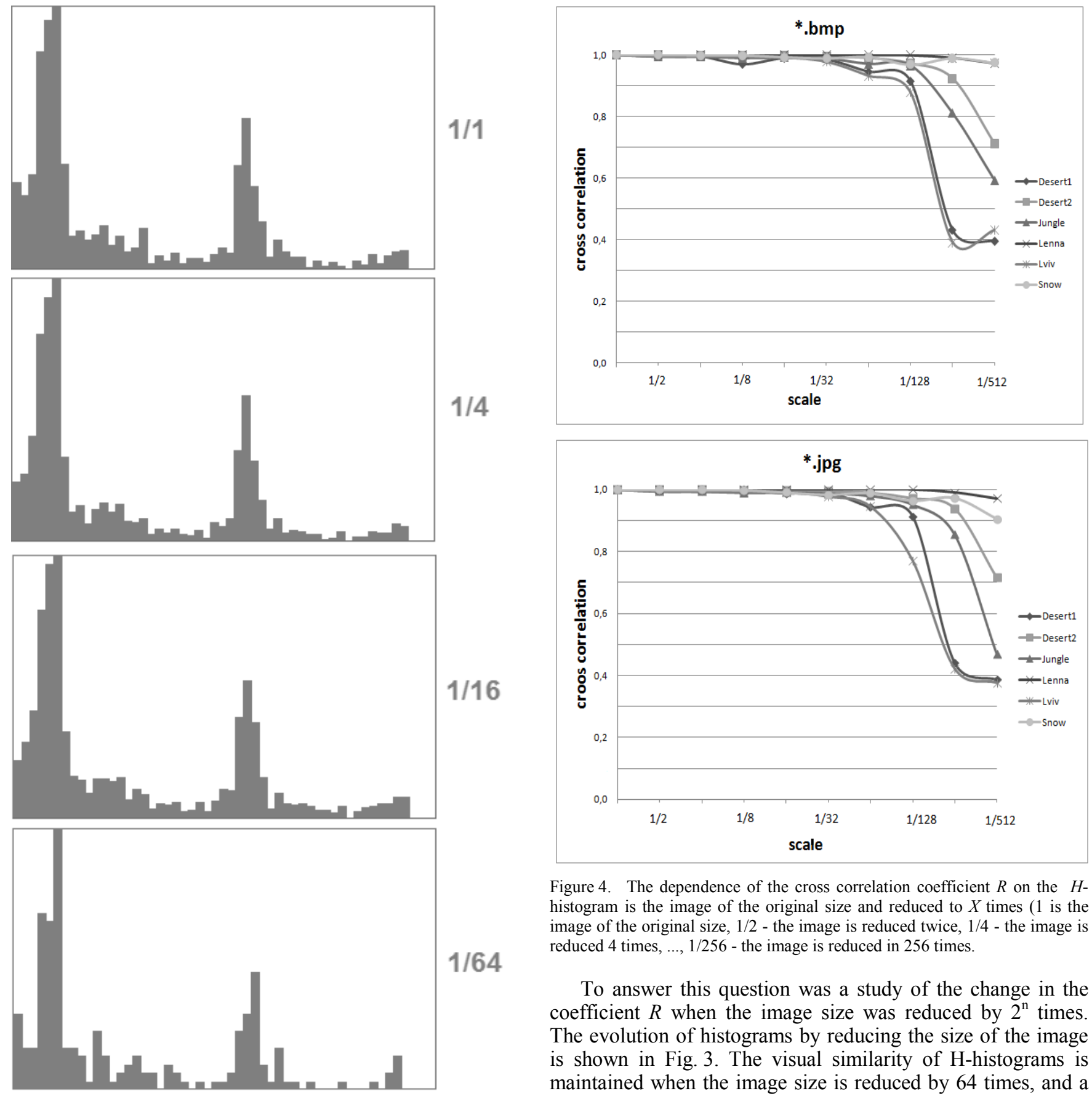

Figure 4. The dependence of the cross correlation coefficient $\mathrm{R}$ on the $\mathrm{H}$ histogram is the image of the original size and reduced to $X$ times ( 1 is the image of the original size, $1 / 2$ - the image is reduced twice, $1 / 4$ - the image is reduced 4 times, ..., $1 / 256$ - the image is reduced in 256 times.

To answer this question was a study of the change in the coefficient $R$ when the image size was reduced by $2^{\mathrm{n}}$ times. The evolution of histograms by reducing the size of the image is shown in Fig. 3. The visual similarity of H-histograms is maintained when the image size is reduced by 64 times, and a change in the numerical degree of similarity is given in Fig. 4.

As can be seen from Fig. 4, for both image formats, the value of the cross-correlation coefficient $R$ remains practically constant even until the image is reduced 32-fold. Insignificant is a decrease in $R$ and a 64-fold scaling, but with a further reduction in the size of the image, the fall of the crosscorrelation coefficient $R$ is significant and does not allow comparisons between them. It should be noted that with a reduction of 128 times the visual similarity of the original and reduced images is extremely difficult to set.

Consequently, even when one of the simplest numerical criteria of similarity degree is applied, which is the crosscorrelation coefficient $R$, can be determined by its threshold (or 
range of values), which will allow unambiguously to classify the image according to their histograms, with a change in their size up to 128 times.

Finally, comparing these values with the results presented in Fig. 3, it is safe to assert that the reduction of the size of the images even 128 times will not affect the quality of obtaining the signs of color and, in the future, their recognition. It should also be added that when applying for the description of the signs of $H$-histogram does not matter and the aspect ratio of the image.

\section{CONCLUSION}

The results of the study of the possibility of using $H$ histograms to compare images and, subsequently, recognition of image fragments due to factors that impair the quality of the image and complicate the obtaining of reliable results, indicate the high degree of suitability of this approach for solving the problem of color image recognition in Content Based Image Retrieval. It is shown that when the image is reduced to 64 times, a very high degree of similarity of the H-histogram is maintained, even in cases where visual similarity is established with some difficulties. The presented results point to the prospect of constructing a descriptor of color images or individual objects based on their $H$-histograms.

\section{REFERENCES}

[1] W. Burger, M.J. Burge, "Principles of Digital image processing: Core Algorithms", Springer, 2009

[2] R.C. Gonzalez and R.E. Woods, "Digital Image Processing", 3rd edition, Prentice- Hall, 2007.

[3] Bakker, E., Lew, M.:" Semantic video retrieval using audio analysis". In: International Conference on Image and Video Retrieval, Lecture Notes in Computer Science, Vol. 2383, 2002, Springer, pp.260-267

[4] Pragati Ashok Deole, Rushi Longadge, "Content Based Image Retrieval using Color Feature Extraction with KNN Classification" International Journal of Computer Science and Mobile Computing, Vol.3 Issue.5, May- 2014, pg. 1274-1280.

[5] Y. Rubner, J. Puzicha, C. Tomasi, J.M. Buhmann, "Empirical evaluation of dissimilarity measures for color and texture", Computer Vision and Image Understanding Vol. 84, No.1, 2001, pp. 25-43.

[6] Anjali Sharma, Ajay Kumar Singh, "Color Difference Histogram for Feature Extraction in Video Retrieval". International Journal of Computer Science \& Engineering Technology. Vol. 6 No.11, 2015 pp.637-642.

[7] A.M. Ferman, A.M. Tekalp, and R. Mehrotra, "Robust color histogram descriptors for video segment retrieval and identification," IEEE Transactions on Image Processing, Vol. 11, No. 5, 2002, pp 497-508.

[8] L. Fei-Fei, R. Fergus and P. Perona. "Learning generative visual models from few training examples: an incremental Bayesian approach tested on 101 object categories". IEEE. CVPR 2004, Workshop on GenerativeModel Based Vision. 2004. 